\title{
Practice and problems with gastrostomies
}

\author{
Gill McHattie \\ Southern General Hospital, South Glasgow University Hospitals Division, Glasgow G51 4TF, UK
}

\begin{abstract}
The development of gastrostomy placement has been an important technological advance in the enteral-access field. However, its rapid growth in popularity could be viewed as problematic. The endoscopist or intervention radiologist can no longer act as a technician but requires to follow-up this group of patients in order to determine outcomes that will inform future practice. There has been emphasis on the importance of the multidisciplinary team in informing and assessing patients referred for gastrostomy insertion. Communication between all the professionals caring for a patient and between the carers and the patient allows information to be collated that will determine the benefits and burdens of long-term gastrostomy feeding. At present much of the published experience is limited to the acute care setting. The incidence of complications varies, depending on the investigator's definition of complication and the diagnosis of the patient group. Many reported studies are retrospective, which puts limitations on documentation. Increasing numbers of patients with diverse needs are now being discharged into the community with gastrostomy tubes in place. Whilst the hospital should ensure that written protocols are provided and that all carers involved should have adequate training, it is not unusual for patients to receive mixed messages from the different care teams responsible for their care. In South Glasgow NHS Hospital Division key members of all teams caring for these patients (acute care, community district nurses, learning disabilities team, physical disabilities team and commercial homecare companies) meet regularly to discuss equipment and protocols. The members of this group feel that this approach has improved communication, standardised practice and reduced complications by providing a service that delivers artificial nutrition support but is primarily suited to the patient's disease process.
\end{abstract}

Gastrostomy: Communication: Clinical governance

As a nutrition nurse specialist the author is part of a nutrition support team that functions in a model similar to that proposed by the British Association for Parenteral and Enteral Nutrition (Silk, 1994) at the Southern General Hospital, Glasgow, which has approximately 900 beds, and includes many regional specialities. A total of 171 gastrostomies were inserted in the year ending December 2003. All gastrostomy referrals from the acute sector and the community sector are made to the nutrition nurse specialists, who assess patients before the referral being processed. The choice of route of insertion is predominantly subjective and evenly divided between the radiological method and the endoscopic method. Current practice is audited with a locally-developed tool at $2 \mathrm{~d}$ post insertion and on removal of the primary tube. Followup care is provided either at disease-specific outpatient clinics or by the district nursing service, which has the back-up of an educational service provided by a community enteral-feeding team.

Assuming a background knowledge of the subject of gastrostomies, problems associated with them and an ability to network with other specialists on this topic, the present paper has taken a more questioning route in examining practice in the twenty-first century and identifying a problem commonly found across the acute and community settings that could be corrected to improve patient care.

\section{Practice}

Ethical and evidence-based practice has come to be associated with modern medicine and is considered in

\footnotetext{
Abbreviation: PEG, percutaneous endoscopic gastrostomy.

Corresponding author: Gill McHattie, fax +44 141201 2999, email Gill.mchattie@sgh.scot.nhs.uk
} 
terms of clinical governance (i.e. 'a framework through which NHS [National Health Service] organisations are accountable for continually improving the quality of their services and safeguarding high standards of care by creating an environment in which excellence in clinical care will flourish'; Scally \& Donaldson, 1998). Tschudin (2003) describes an ethical approach to care as one based on a relationship between the individual giving care and the individual receiving care. Care has to be appropriate and adequate and should consider the needs of the recipient. This care then requires the caregiver to be competent and knowledgeable. Literature on the practice of gastrostomy insertion and follow-up care has been widely available over the last 20 years.

\section{Inconsistent practice}

Bowling (2002) has reported on the British Society of Gastroenterologists' questionnaire, which involved a postal survey of consultants in the endoscopy units of 242 British hospitals that had a response rate of $81 \%$. The results show inconsistencies of practice, with $25 \%$ of gastroenterologists viewing themselves as technicians and not assessing the patient before percutaneous endoscopic gastrostomy (PEG) insertion. Only $7 \%$ of the hospitals were reported to offer any kind of multidisciplinary follow up to PEG insertion. Inconsistencies of practice have also been reported by the National Confidential Enquiry into Patient Outcome and Death (2004), an organisation that operates under the umbrella of the National Institute of Clinical Excellence. Their report indicates a mortality of $6 \%$, equating to 986 patients of the 16648 who had PEG procedures in 2003. Of these procedures $19 \%$ were considered to be futile by the advisors to the National Confidential Enquiry into Patient Outcome and Death. Interestingly, it was also found that in $42 \%$ of these procedures the patients had received no prophylactic antibiotics despite the recommendation from the British Society of Gastroenterology (1996).

\section{Disease-specific practice}

Evidence of the benefits of gastrostomy insertion in specific diseases is sparse and difficult to interpret because of the debilitation of the underlying disease (DeLegge, $2003 b$ ). Gauderer (2002) has described the evolution of the PEG designed to benefit children with impaired swallowing. There was early recognition by physicians in the 1980s that this longer-term route of access for nutritional support would benefit patients with stroke disease. The Scottish Intercollegiate Guidelines Network (2004) clinical guideline on the management of patients with stroke states that the decision to place a PEG should balance the risks and benefits, and quotes the findings of the FOOD (feed or ordinary diet) Trials of The International Stroke Trials Collaboration (see www.dcn.ed.ac.uk/food) that early tube feeding should be via a naso-gastric tube. The recommendation for gastrostomy insertion in patients with motor neurone disease (Leigh et al. 2003) is that it should take place early in the disease process before vital capacity falls. Leigh et al. (2003) have concluded that a radiologically-inserted gastrostomy may have advantages over a PEG. Experience with motor neurone disease at the Southern General Hospital centre is anecdotal; 14\% of gastrostomy insertions in 2003 were for patients with motor neurone disease, often late in their diagnostic journey. The appropriateness of this approach is debated but care must be taken that the multidisciplinary team do not become paternalistic in judging quality of life.

The evidence produced in the last 10 years (Tyldesley et al. 1996; Lee et al. 1998; Scolapio et al. 2001; Tucker et al. 2003) supports the use of prophylactic gastrostomy insertion for patients with head and neck cancer to minimise weight loss and reduce interruptions in treatment programmes. This evidence is reflected in practice, with the British Artificial Nutrition Survey (Glencorse et al. 2003) showing a point prevalence rise from ten in 1996 to 866 in 2002 for patients with head and neck cancer. The diagnosis of head and neck cancer accounts for $39 \%$ of gastrostomy insertions in 2003 in the South Glasgow NHS Hospital Division.

\section{Type of practice}

There is no specific evidence that can be used in deciding which method of gastrostomy insertion to employ; much of the literature is retrospective and not comparative. Muller et al. (1999) have found in their retrospective comparative study that there is no statistical difference between the three methods of gastrostomy insertion in relation to complication rates. Prospective work is required to provide an evidence-based practice, when referring patients for particular methods of gastrostomy insertion, to allow patients to make an informed choice.

\section{Problems}

A great deal of literature has been produced to inform practice of the complications associated with endoscopic insertion of gastrostomy tubes. These complications are well documented, most recently by DeLegge (2003a) and Stroud et al. (2003). Hospitals, nursing homes and primary care settings are required to develop education and guidelines for the carers of patients with gastrostomy tubes in situ that attempt to avoid these complications where possible. Practices surrounding the insertion of radiological gastrostomies and complications associated with this procedure are not as well documented and require studies to be carried out and published.

\section{Problem of accidental tube removal}

The complication of accidental tube removal can be the most immediate emergency presented to the community staff. Education and availability of replacement gastrostomy tubes of the correct size is important. The Medicines and Healthcare Products Regulatory Agency (2004) have stated that Foley catheters are not an acceptable replacement tube. Good communication with the patient and carers to ensure that they are aware of where to go for assistance is essential. 


\section{Problem of communication}

In South Glasgow NHS Hospital Division there are many different teams referring and caring for the patient on gastrostomy nutritional support (acute care, community district nurses, learning disabilities team, physical disabilities team and commercial homecare companies). It has been found that quarterly meetings of the key members of these teams have opened dialogue, established standardised practice and equipment and shared knowledge. This informal group approach appears to have improved care, and certainly in the acute setting there have been fewer crisis management phone calls. Patients remain with an appropriate healthcare team rather than a nutrition team and a core team.

\section{Problems should inform practice; should reduce problems!}

Artificial nutrition and hydration has traditionally been used to meet therapeutic goals; however, there are some patient populations that may be at an increased risk of complications as a result of this treatment (DeLegge 2003a). Thus, it is pertinent to consider clinical benefits and burdens before commencing, withholding or withdrawing this treatment. In order to understand the potential benefits the healthcare team must be aware of current evidence to support the information given to the patient in order to allow them to make an informed choice. The healthcare team must consult widely and there must be communication between those members of the team working in the acute setting and those members working in community settings. Both groups have different skills and knowledge to share and contribute to decisions that affect the quality of care delivered to the patients.

\section{References}

Bowling TE (2002) UK PEG Service: Are we really practising in the patients' best interests? Clinical Nutrition Update 7, 6-8.

British Society of Gastroenterology (1996) Antibiotic Prophylaxis in Gastrointestinal Endoscopy. London: British Society of Gastroenterology.

DeLegge MH (2003a) Prevention and Management of Complications from Percutaneous Endoscopic Gastrostomy. Wellesley, MA: UpToDate.

DeLegge MH (2003b) PEG Placement: Justifying the Intervention. Wellesley, MA: UpToDate.
Gauderer MWL (2002) Percutaneous endoscopic gastrostomy and the evolution of contemporary long-term access. Clinical Nutrition 21, 103-110.

Glencorse C, Meadows N \& Holden C (editors) (2003) BANS Report: Trends in Artificial Nutrition Support in the UK between 1996 and 2002. Reddich, Worcs.: BAPEN.

Lee JH, Machtay M, Unger LD, Weinstein GS, Weber RS, Chalian AA \& Rosenthal DI (1998) Prophylactic gastrostomy tubes in patients undergoing intensive irradiation for cancer of the head and neck. Archives of Otolaryngology - Head and Neck Surgery 124, 871-875.

Leigh PN, Abrahams S, Al-Chalabi A, Ampong M-A, Goldstein LH, Johnson J et al. (2003) The management of motor neurone disease. Journal of Neurology, Neurosurgery, and Psychiatry 74, 32-47.

Medicines and Healthcare Products Regulatory Agency (2004) Safety Action Notice SAN(SC)04/03-11 Feb 2004. Misuse or Modification of Medical Devices/Non-medical Products: Safety, Suitability and Effectiveness Issues. London: Department of Health.

Muller P, Lindberg CG \& Zilling T (1999) Gastrostomy by various techniques: evaluation of indications, outcome and complications. Scandinavian Journal of Gastroenterology $\mathbf{1 0}$, $1050-1054$.

National Confidential Enquiry into Patient Outcome and Death (2004) Scoping our practice. http://www.ncepod.org.uk/ 2004report/index.htm

Scally G \& Donaldson LJ (1998) British Medical Journal 317 61-65.

Scolapio JS, Spangler PR, Romano MM, McLaughlin MP \& Salassa JR (2001) Prophylactic placement of gastrostomy feeding tubes before radiotherapy in patients with head and neck cancer: is it worthwhile? Journal of Clinical Gastroenterology 33, 215-217.

Scottish Intercollegiate Guidelines Network (2004) Management of Patients with Stroke: Identification and Management of Dysphagia. Clinical Guideline no. 78. Edinburgh: Royal College of Physicians; available at http://www.sign.ac.uk/pdf/ sign78.pdf

Silk DBA (editor) (1994) Organisation of Nutritional Support in Hospitals. Reddich, Worcs.: BAPEN.

Stroud M, Duncan H \& Nightingale J (2003) Guidelines for enteral feeding in adult hospital patients. Gut 52, Suppl. 7, vii1-vii12.

Tschudin V (2003) Ethics in Nursing: The Caring Relationship, 3rd ed. Edinburgh: Butterworth Heineman.

Tucker AT, Gourin CG, Ghegan MD, Porubsky ES, Martindale RG \& Terris DJ (2003) 'Push' versus 'pull' percutaneous endoscopic gastrostomy placement in patients with advanced head and neck cancer. Laryngoscope 113, 1898-1902.

Tyldesley S, Sheehan F, Munk P, Tsang V, Skarsgard D, Bowman C \& Hobenshield SE (1996) The use of radiologically placed gastrostomy tubes in head and neck cancer patients receiving radiotherapy. International Journal of Radiation Oncology Biology and Physics 36, 1205-1209. 
https://doi.org/10.1079/PNS2005440 Published online by Cambridge University Press 